\title{
Translational and Rotational Diffusion during Radical Bulk Polymerization: A Comparative Investigation by Full Correlation Fluorescence Correlation Spectroscopy (fcFCS)
}

\author{
Maren Dorfschmid, ${ }^{\dagger}$ Klaus Müllen, ${ }^{\ddagger}$ Andreas Zumbusch, ${ }^{\dagger}$ and Dominik Wöll ${ }^{*} \dagger, \S$ \\ ${ }^{\dagger}$ Fachbereich Chemie, Universität Konstanz, Universitätsstrasse 10, 78464 Konstanz, Germany, \\ ${ }^{\S}$ Zukunftskolleg, Universität Konstanz, Universitätsstrasse 10, 78464 Konstanz, Germany, and \\ Max-Planck-Institut für Polymerforschung, Ackermannweg 10, 55128 Mainz, Germany
}

Received April 21, 2010; Revised Manuscript Received June 17, 2010

\begin{abstract}
Diffusional changes during the radical bulk polymerization of styrene with and without crosslinker were investigated. Full correlation fluorescence correlation spectroscopy (fcFCS) allowed for the simultaneous detection of rotational and translational diffusion of single fluorescent perylene diimide probes. Small probes show a parallel decrease of both motions whereas for larger probes translation slows down faster than rotation. The latter behavior contradicts the relationship between the Stokes-Einstein and the Stokes-Einstein-Debye equation and is attributed to changes in the microviscosity and mesh size effects of the synthesized polymer chains. The introduction of permanent cross-links results in additional restrictions for translational diffusion whereas rotation is not significantly affected up to high monomer-to-polymer conversions.
\end{abstract}

\section{Introduction}

Diffusion and dynamics in polymers and polymer solutions have attracted many researchers for a long time. ${ }^{1-4}$ The interest in diffusion not only stems from questions of fundamental research but also is crucial for designing and evaluating separation and drug-delivery systems. In particular, during bulk radical polymerization, evolving diffusional limitations determine polymerization kinetics 5 and thus the properties of the produced polymer such as the molecular mass distribution. Impressive progress to relate polymer physics to chemical kinetics has been achieved by several groups. ${ }^{6-9}$ However, many questions about this complex relationship still remain open. Despite efforts to understand diffusion in polymer solutions, there is no universally valid theory which could describe mobility from highly dilute to highly concentrated polymer solutions. In general, the translational diffusion coefficient $D_{\mathrm{T}}$ can be obtained from the StokesEinstein (SE) relation $D_{\mathrm{T}}=k T / 6 \pi \eta r_{\mathrm{h}}$, which requires knowledge of the hydrodynamic radius $r_{\mathrm{h}}$ and the viscosity $\eta$. Knowing the diffusion coefficient of a molecule, the SE relation allows for the determination of the local viscosity which affects its velocity, the so-called microviscosity. The investigation of the diffusion of guest molecules in polymer solutions has been achieved using many different techniques. Most popular are field-gradient NMR, ${ }^{10,11}$ fluorescence recovery after photobleaching (FRAP), ${ }^{12}$ forced Rayleigh scattering, ${ }^{11,13,14}$ Taylor dispersion, and phosphorescence quenching measurements. ${ }^{15}$ Comparison of the diffusion data with models shows that a reliable theory which describes or even predicts the diffusion in different polymer solutions over an extended concentration range does not yet exist. Despite this lack of a universal model, there have been powerful approaches which within their assumptions and approximations yield good agreement with the experimental observations. The most prominent models are based on free volume theory ${ }^{16}$ and hydrodynamic theory. ${ }^{17}$ The models for diffusion in polymer

*Corresponding author. E-mail: dominik.woell@uni-konstanz.de. solutions have been reviewed by Masaro and Zhu. ${ }^{18}$ The main objective behind these approaches is to find appropriate scaling laws for important quantities, ${ }^{19}$ one of them being the diffusion coefficient, and to get clear ideas of the microscopic dynamics determining the properties of these systems.

Fluorescence correlation spectroscopy $(\mathrm{FCS})^{20,21}$ has been extensively used to determine the diffusion coefficients in polymer solutions, ${ }^{22-24}$ polymer networks, ${ }^{25}$ polymer melts, ${ }^{26}$ and during bulk radical polymerization ${ }^{27}$ and the diffusion of labeled polystyrene chains in different solvents ${ }^{28}$ and polymer solutions s $^{23,29,30}$ for analyzing diffusion in polymer films well above the glass transition temperature ${ }^{31}$ and for the diffusion of gold nanoparticles in semidilute and concentrated polystyrene solutions. ${ }^{32}$ Since the diffusion of molecules is directly related to the microviscosity of their surrounding, FCS has also been proven to be a suitable method for microrheological measurements. ${ }^{33}$

To date, most FCS investigations have been limited in temporal resolution to ca. $0.1 \mu \mathrm{s}$, the avalanche photodiode (APD) dead time. Thus, only translational diffusion and triplet blinking ${ }^{34}$ could be analyzed; faster processes remained obscured. In order to acquire shorter correlation times, two APDs arranged in a Hanbury Brown and Twiss setup ${ }^{35}$ are required. Cross-correlation of the signals acquired on both APDs results in full correlation FCS curves. ${ }^{36,37}$ These cross-correlation curves contain information about rotational diffusion and antibunching. ${ }^{38,39}$ Antibunching results in a drop in correlation observed at short times if only a single fluorophore emits at a given time. In this case the time interval between two emitted photons is determined by the excitation frequency and the fluorescence lifetime of the fluorophore.

A theory on the analysis of rotational diffusion from FCS measurements was developed by Ehrenberg/Rigler ${ }^{40}$ and Aragón/ Pecora. ${ }^{41,42}$ Several years later, Kask et al. were the first to experimentally observe rotational motion and to estimate the rotational diffusion coefficient of fluorescently labeled bovine carbonic anhydrase B by FCS. ${ }^{43}$ The same group determined 
Scheme 1. Dyes $(1-3),{ }^{47}$ Initiator (4), and Cross-Linker (5) Used in This Study

$\mathbf{R}=$<smiles></smiles>

1<smiles>[R]c1ccc(-c2cc(-c3ccccc3)c(-c3ccc(-c4cc(-c5ccccc5)c(-c5ccccc5)c(-c5ccccc5)c4-c4ccccc4)cc3)c(-c3ccccc3)c2-c2ccccc2)c(-c2ccccc2)c1-c1ccccc1</smiles>

2

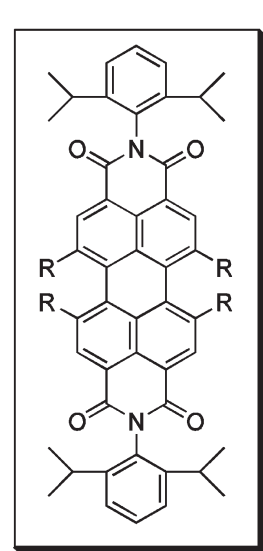

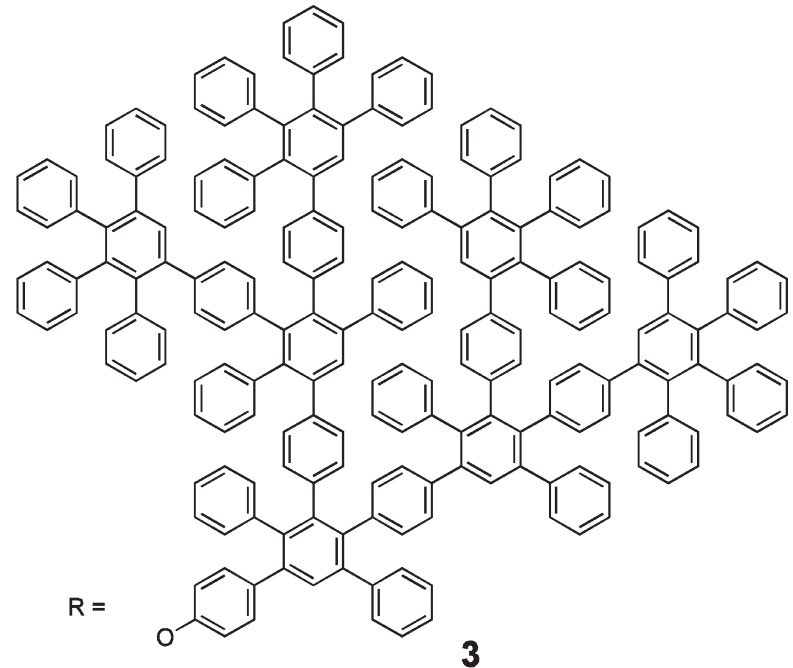

3<smiles>COC(C)(C)CC(C)(C)N=NC(C)(C)CC(C)(C)C</smiles>

how the polarization of excitation and emitted light changes the amplitude of the rotational contribution to the correlation function. Thus, they could optimize the conditions to measure rotational motion. ${ }^{44}$

The possibility to record full correlation FCS data and thus to observe rotational and translational diffusion in the same system at the same time grants access to fundamental information about the dynamics of molecules. Tsay et al. have demonstrated the feasibility of the method to observe rotational and translational diffusion of peptide-coated nanorods in media of different viscosities. ${ }^{45}$ Furthermore, an interleaved excitation scheme with crossed excitation polarization to maximize the amplitude of the contribution of rotation in the correlation curve has been suggested by Loman et al. ${ }^{46}$ However, full correlation FCS has not been used so far for the observation of translation and rotation in polymer systems.

In this work, we show that full correlation fluorescence correlation spectroscopy ( $\mathrm{fcFCS}$ ) can be utilized for a parallel investigation of translational and rotational motion of single fluorescent perylene diimide molecules during the radical bulk polymerization of styrene. Thus, we can observe how the increasing density of polymer chains affects rotational and translational diffusion during polymerization. To the best of our knowledge, this is the first reported observation of rotation by fcFCS over an extended range of rotational diffusion coefficients. Dyes of different sizes were investigated in order to carefully assess size effects of the molecules on their mobility, and the effect of crosslinking on diffusion was investigated.

\section{Experimental Methods}

Chemicals. Styrene (99\%, Merck) was distilled under reduced pressure before use. The radical initiator V70 (Wako Chemicals, 2,2'-azobis(4-methoxy-2,4-dimethylpentanenitrile), 4, Scheme 1), 1,4-divinylbenzene (Sigma-Aldrich), and toluene (99.5\%, SigmaAldrich) were used without further purification. The perylene diimide dyes (see Scheme 1) were synthesized in the Müllen group at the MPI for Polymer Research, Mainz. Their synthesis is described elsewhere. ${ }^{47}$

Polymerizations. In a typical polymerization experiment, $1 \%$ $(\mathrm{w} / \mathrm{w})$ of thermal radical initiator V70 was dissolved in styrene in a Schlenk tube which was plasma cleaned before. For the polymerizations with cross-linker, $1 \%(\mathrm{w} / \mathrm{w})$ of 1,4-divinylbenzene 5 was additionally added. The dye was dissolved in toluene and diluted several times until an appropriate concentration $(\sim 1 \mathrm{nM})$ was reached. One drop of this dye solution (less than $1 \% \mathrm{w} / \mathrm{w}$ ) was then added to the Schlenk tube with polymerization solution. Oxygen was removed by three freeze-pumpthaw cycles. In a glovebox, the solution was refilled into homebuilt closed sample holders for microscopy containing a small stirring bar. All polymerizations were stirred as long as possible until the viscosity of the polymerization mixture became too high. They were performed at room temperature which resulted in a rather moderate conversion rate allowing extensive FCS measurements at each conversion.

FCS Measurements. The beam of a cw-DPSS laser (532 nm, Cobolt Samba) was optimized to an approximate Gaussian shape by several lenses, attenuated to ca. $150 \mu \mathrm{W}$ by neutral density filters, and vertically polarized, coupled into an optical microscope (Zeiss, Axiovert 200) with a water immersion objective (Zeiss, LCI Plan-Neofluar, $63 \times$ magnification, NA $=1.3$ ) to excite the molecules in the confocal volume with the center at a distance of $10 \mu \mathrm{m}$ from the slide surface. Their fluorescence was collected by the same objective and passed through a $40 \mu \mathrm{m}$ pinhole in order to reject light from out-of-focus regions. Scattered excitation light was removed by a dichroic mirror (z532 RDC, Chroma) before the pinhole and a notch filter (NF01-532U-25, Semrock) and a long pass filter (LP568, Semrock) after the pinhole. In order to increase the contrast of the rotational contribution to the autocorrelation, the fluorescence light was sent through a polarizer set to horizontal polarization which in our setup corresponds to the polarization of the excitation beam. ${ }^{44,45}$ It was divided onto two avalanche photodiodes (APDs: SPCM-AQR-14, Perkin-Elmer) by a 50:50 beam splitter (03BSC007, Melles Griot). Detection with two APDs in a Hanbury Brown and Twiss setup ${ }^{35}$ and crosscorrelation of their signals allows for detection at times shorter than the deadtime of each APD and removal of APD afterpulses. In order to reduce cross-communication between both APDs by breakdown flashes, ${ }^{48}$ a short pass filter (ET 680SP$2 \mathrm{P} 8$, Chroma) was placed in front of one APD and a bandpass filter (FF01-593/40-25, Semrock) in front of the other. The count rates on each APD were about 3000 counts/s. Measurements were typically performed for $20 \mathrm{~min}$, thus collecting altogether ca. 7 million photons. Each photon event was 


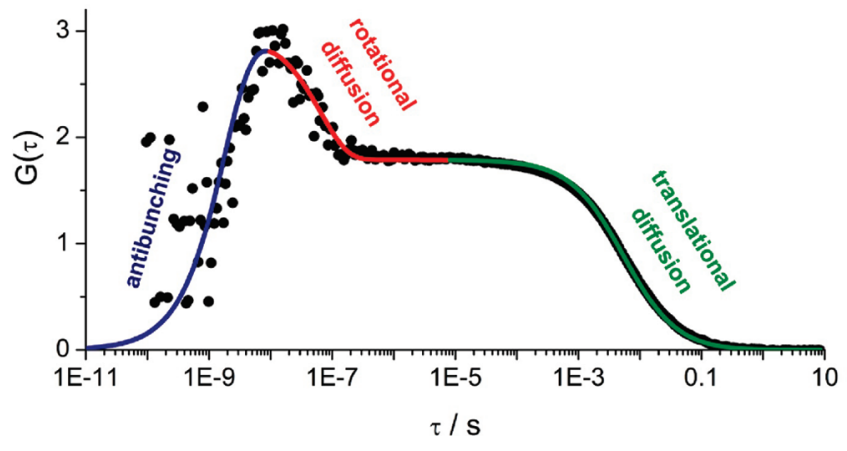

Figure 1. Representative fcFCS correlation curve for dye $\mathbf{1}$ during the polymerization of styrene at $80 \%$ conversion. Contributions due to antibunching, rotational, and translational diffusion are indicated.

recorded with a time stamp with picosecond precision by a Hydraharp 400 (Picoquant, Berlin) in T2 mode. Cross-correlations between the photon events of both APDs were calculated by the Symphotime software (Picoquant) and yielded full correlation $\mathrm{FCS}^{36,37}$ (fcFCS) curves.

Dyes. The synthesis of the dyes is described in the literature. ${ }^{47}$ Their structure was optimized and their hydrodynamic radii calculated using the MM2 package within ChemDraw 3D Ultra. The radii of the dyes were determined to be $10 \AA$ for dye $\mathbf{1}, 24 \AA$ for dye $\mathbf{2}$, and $32 \AA$ for dye 3 (Scheme 1).

\section{Results}

Determination of Diffusional Coefficients. Compared to "classical" FCS measurements with one APD, fcFCS allows for an extension of the time range into the nano- to picoseconds range. A typical fcFCS correlation curve is shown in Figure 1. Herein, three gradual changes of the correlation function can be differentiated corresponding to antibunching $\left(<10^{-8} \mathrm{~s}\right)$, rotational $\left(10^{-8}-10^{-6} \mathrm{~s}\right)$, and translational motion $\left(10^{-5}-1 \mathrm{~s}\right)$. None of our correlation curves exhibited contributions of intersystem crossing. Compared to other dyes, the ISC rate of the perylene diimides used seems to be too low for triplet blinking to give significant contributions. This is a considerable advantage especially when rotational diffusion is expected to be on a similar time scale because otherwise it would be very hard to separate these processes and fit the corresponding curves.

In order to determine the rotational and translational diffusion coefficient, the correlation curve has to be fitted using an appropriate model. Including rotational contributions into the correlation function is rather complex for the general case. ${ }^{42,44}$ However, if the processes appearing in the correlation curve are well separated in time, the correlation function can be simplified by factorization. ${ }^{21,49}$ For the polarizer settings and the geometry used in our experiments, Aragón and Pecora ${ }^{41}$ have shown that the rotational contribution is proportional to $80 \mathrm{e}^{-6 D_{\mathrm{R}} t}+$ $(64 / 9) \mathrm{e}^{-20 D_{\mathrm{R}} t}$, if the fluorescence lifetime $\tau_{\mathrm{f}}$ is much shorter than the rotational diffusion time $\tau_{\mathrm{R}}$. Herein, the two terms correspond to solutions of the diffusion equation for spherical diffusers with the eigenvalues $l(l+1)$ of the angular momentum operator $\mathbf{L}^{2}$ with quantum numbers $l=2$ or $l=4$, respectively. The amplitude of the second term is $\sim 1$ order of magnitude lower than that of the first term and thus will be neglected throughout this paper.

For the overall fit of the autocorrelation function, we used the following equation:

$G(\tau)=\frac{1}{N}\left(1-\mathrm{e}^{-\tau / \tau_{\mathrm{AB}}}\right)\left(1+A \mathrm{e}^{-\tau / \tau_{\mathrm{R}}}\right)\left(1+\frac{\tau}{\tau_{\mathrm{D}}}\right)^{-1}\left(1+\frac{\tau}{\omega^{2} \tau_{\mathrm{D}}}\right)^{-1 / 2}$
Herein, $N$ is the average number of molecules in the confocal volume, $A$ is a factor representing the rotational contribution, $\omega$ is a geometric factor of the confocal volume, $\tau_{\mathrm{AB}}$ is the average time of antibunching, and $\tau_{\mathrm{R}}$ and $\tau_{\mathrm{D}}$ are the correlation times for rotational and translational diffusion, respectively. From the correlation times, the rotational and translational diffusion coefficients were calculated according to

$$
D_{\mathrm{R}}=\frac{1}{6 \tau_{\mathrm{R}}}
$$

and

$$
D_{\mathrm{T}}=\frac{\left\langle x^{2}\right\rangle}{4 \tau_{\mathrm{D}}}
$$

where $\left\langle x^{2}\right\rangle$ was determined by a reference measurement (Rhodamine $6 \mathrm{G}$ in water with a reference diffusion coefficient ${ }^{50}$ of $4.14 \times 10^{-10} \mathrm{~m}^{2} \mathrm{~s}^{-1}$ ).

Rotational and Translational Diffusion Coefficients during Linear Polymerization. During the free radical bulk polymerization of styrene without cross-linker, we recorded fcFCS curves for all three dyes at regular intervals. Three representative curves for dye 3 are shown in Figure 2. In all curves, the contributions due to antibunching, rotational, and translational motion can be clearly observed. At low correlation times, significant scattering of the data points is observed due to decreasing width of the time-binning intervals. With increasing monomer conversion, both the translational and the rotational diffusion times become longer. All curves were fitted using eq 1 , and the diffusion coefficients were calculated from the diffusion times using eqs 2 and 3 . Rotational (Figure 3a) and translational (Figure 3b) diffusion coefficients were plotted versus monomer-to-polymer conversion and polymerization time, respectively. For the determination of the conversion from the polymerization time we performed Raman measurements (see Supporting Information).

The evolution of rotational diffusion coefficients $D_{\mathrm{R}}$ for all three dyes used during the radical polymerization of styrene is shown in Figure 3a. For a better comparison of the relative changes of $D_{\mathrm{R}}$, the data were normalized to their value in pure styrene solution (at a conversion of $0 \%$ ). The initial absolute values for $D_{\mathrm{R}}$ are $3.8 \times 10^{8} \mathrm{~s}^{-1}$ for dye $1,2.5 \times$ $10^{7} \mathrm{~s}^{-1}$ for dye 2 , and $1.1 \times 10^{7} \mathrm{~s}^{-1}$ for dye 3 . In addition to fcFCS, we used fluorescence anisotropy measurements $(\mathrm{FA})^{51}$ to yield data points at low conversions where, in fcFCS, correlation due to rotational motion is obscured by antibunching (see Figure 3a). FA, on the other hand, is restricted to time scales in the order of the fluorescence lifetime, i.e., to rotation measurements at low conversions. Thus, both methods, fcFCS and FA measurements, complement each other to obtain rotational diffusion coefficients over a large conversion range. The normalized rotational diffusion coefficients lie on a master curve within the entire conversion range, i.e., relative changes in rotational diffusion are independent of the size of the dye.

In contrast to the observations of rotational diffusion, the relative changes of the translational diffusion coefficient depend on the size of the dye as shown in Figure 3b. With increasing size the slope of the normalized $D_{\mathrm{T}}$ versus conversion becomes steeper. The shapes of the curves resemble each other with a rather shallow slope for conversions below $25 \%$ followed by a steeper slope up to a conversion of $40 \%$. The diffusion coefficients of all dyes drop rapidly at a conversion over $80 \%$. The translational diffusion coefficients $D_{\mathrm{T}}$ possess initial values of $4.4 \times 10^{-10} \mathrm{~m}^{2} \mathrm{~s}^{-1}$ for 

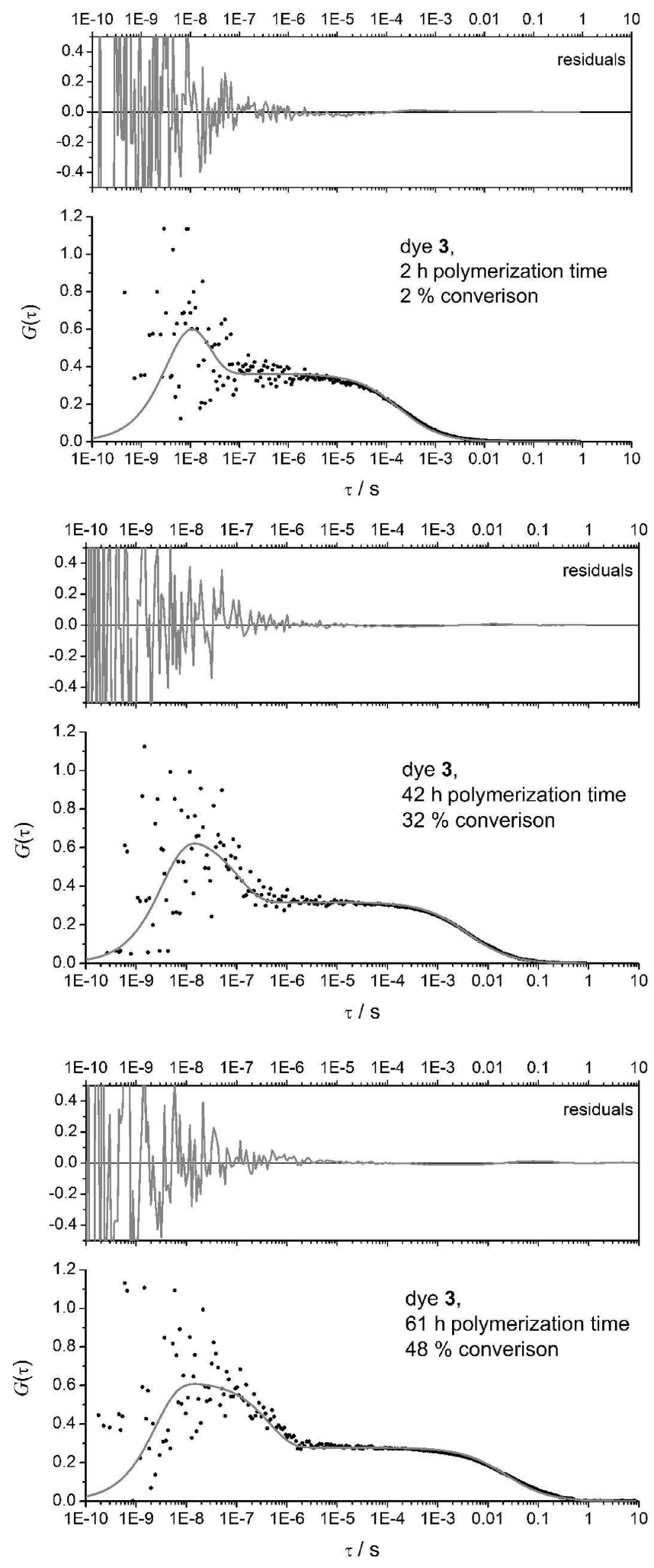

Figure 2. Autocorrelation curves of the fluorescence intensity traces of dye $\mathbf{3}$ during the free radical polymerization of styrene. The data points (full circles) were fitted according to eq 1 (gray line).

dye $1,2.8 \times 10^{-10} \mathrm{~m}^{2} \mathrm{~s}^{-1}$ for dye 2 , and $1.7 \times 10^{-10} \mathrm{~m}^{2} \mathrm{~s}^{-1}$ for dye 3 .

Rotational and Translational Diffusion Coefficients during Cross-Linking Polymerization. In order to analyze differences between systems of linear and covalently cross-linked chains, we performed a cross-linking polymerization with
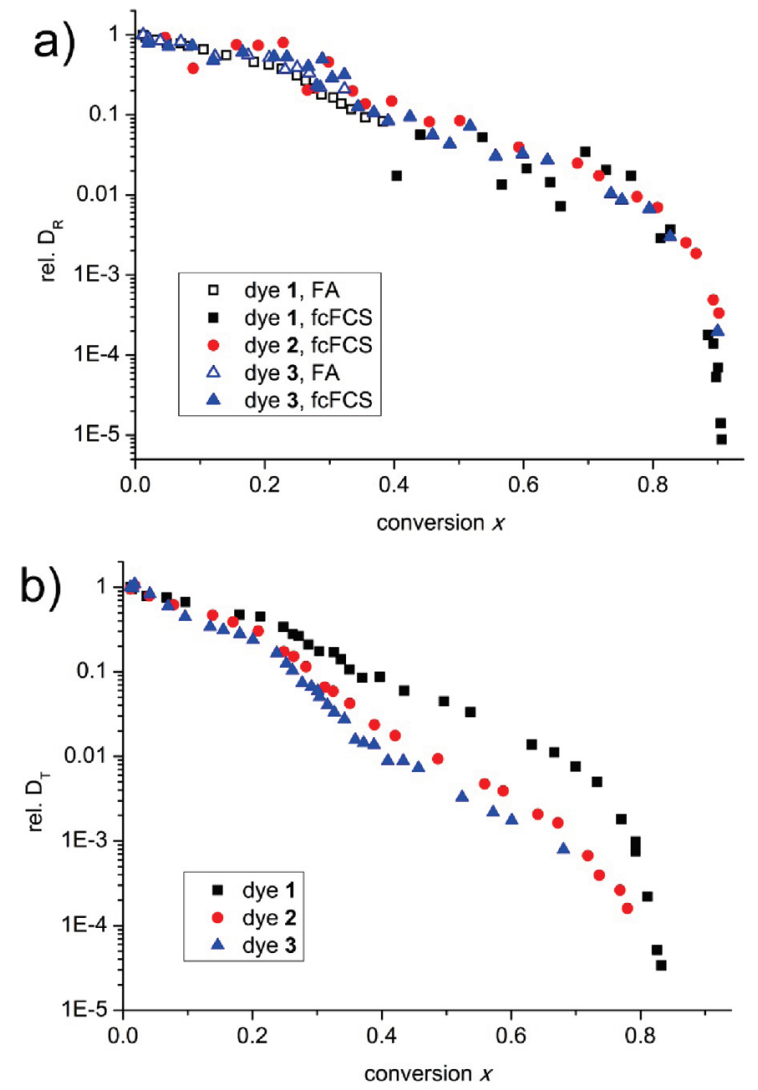

Figure 3. Relative changes of rotational (a) and translational (b) diffusion coefficients during the free radical polymerization of styrene (without cross-linker).

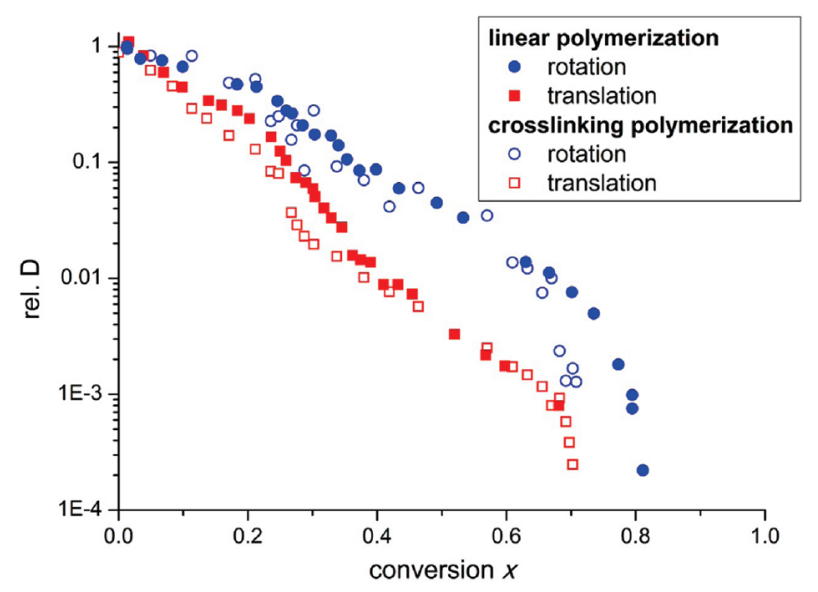

Figure 4. Comparison of relative changes in translational (circles) and rotational (squares) diffusion between linear (full symbols) and crosslinking (open symbols) radical bulk polymerization of styrene using dye 3 as probe.

$1 \%(\mathrm{w} / \mathrm{w})$ of cross-linker and dye 3 as probe (Figure 4$)$. It could be observed that, up to a conversion of 0.65 , rotational motion behaves similarly to the corresponding polymerizations without cross-linker. The translational diffusion coefficients however are slightly lower in the presence of crosslinker which indicates additional restrictions in translational mobility due to permanent cross-links. The differences in translational diffusion are most pronounced at conversions around $30 \%$ where they reach a factor of ca. 2. At conversions larger than $50 \%$, no differences in translation during linear and cross-linking polymerization were found. 
Table 1. Calculation of $r_{\mathrm{h}}$ by a Comparison between Translational and Rotational Diffusion Coefficient

\begin{tabular}{|c|c|c|c|c|}
\hline & \multirow[b]{2}{*}{$D_{\mathrm{T}} / 10^{-10} \mathrm{~m}^{2} \mathrm{~s}^{-1}$} & \multirow[b]{2}{*}{$D_{\mathrm{R}} / 10^{6} \mathrm{~s}^{-1}$} & \multicolumn{2}{|c|}{$r_{\mathrm{h}} / 10^{-9} \mathrm{~m}$} \\
\hline & & & $\begin{array}{c}\text { from } \\
\text { measurement }\end{array}$ & $\begin{array}{l}\text { from } \\
\text { modeling }\end{array}$ \\
\hline dye 1 & 4.4 & 380 & 0.9 & 1.0 \\
\hline dye 2 & 2.8 & 25 & 2.9 & 2.4 \\
\hline dye 3 & 1.7 & 11 & 3.4 & 3.2 \\
\hline
\end{tabular}

\section{Discussion}

Translational and rotational diffusion can be analyzed using the Stokes-Einstein (SE, eq 4) and the Stokes-Einstein-Debye equation (SED, eq 5), respectively.

$$
\begin{gathered}
D_{\mathrm{T}}=\frac{k_{\mathrm{B}} T}{6 \pi \eta r_{\mathrm{h}}} \\
D_{\mathrm{R}}=\frac{k_{\mathrm{B}} T}{8 \pi \eta r_{\mathrm{h}}{ }^{3}}
\end{gathered}
$$

Here, $k_{\mathrm{B}}$ is the Boltzmann constant, $T$ the temperature, $\eta$ the viscosity, and $r_{\mathrm{h}}$ the hydrodynamic radius of the diffusing molecule. If these equations are valid, a comparison between rotational and translational diffusion allows for calculation of the hydrodynamic radius $r_{\mathrm{h}}$ as follows:

$$
r_{\mathrm{h}}=\sqrt{\frac{3 D_{\mathrm{T}}}{4 D_{\mathrm{R}}}}
$$

Table 1 summarizes the hydrodynamic radii obtained for dyes $\mathbf{1}, \mathbf{2}$, and $\mathbf{3}$ making use of the translational and rotational diffusion coefficient in styrene solution (at $0 \%$ conversion). The radii obtained are in good agreement with the values estimated by molecular modeling.

Both the SE and the SED equations predict that the diffusion coefficient is inversely proportional to viscosity. Thus, if these equations hold, both translational and rotational diffusion are expected to exhibit the same relative changes when varying viscosity, i.e., for increasing conversion, and the ratio between $D_{\mathrm{R}}$ and $D_{\mathrm{T}}$ should remain constant. Also, when dyes of different sizes are compared to each other, the relative changes should not differ because viscosity is not expected to depend on the size of the probe. However, the Stokes-Einstein (-Debye) equation is only valid for probes in homogeneous media, and thus it is expected that deviations will occur with increasing heterogeneity of the system. It was the purpose of this study to show if the same deviations can be observed for translational and rotational diffusion or if, at some point, the respective motions exhibit different behavior. In order to elucidate this point, we calculated the ratio of the rotational and translational diffusion coefficient and normalized it to the ratio of the motions in pure monomer solution, a situation for which the Stokes-Einstein and the Stokes-Einstein-Debye equation certainly holds:

$$
\frac{D_{\mathrm{R}}}{D_{\mathrm{T}}}: \frac{D_{\mathrm{R}, 0}}{D_{\mathrm{T}, 0}} \equiv \chi
$$

The ratio $\chi$ remains constant at $\chi \approx 1$ for the small dye 1 during the entire course of polymerization, indicating that translational and rotational motion slow down in a similar manner as predicted by the SE and the SED equation. This is obvious from a comparison of Figure 3a,b which show very similar behavior for dye $\mathbf{1}$. For the two larger probes, our observations cannot be simply explained by the viscosity dependence of the diffusion coefficients as described by the SE and SED equation. It could be shown that even at low conversions $<10 \%$ (the conversion at which overlap of the polymer chains occurs was calculated to be $4 \%$ ) translational diffusion decreases faster than expected by rotation. Translational motion of the larger dye $\mathbf{3}$ slows down more than of dye $\mathbf{2}$. However, the difference between small dye $\mathbf{1}$ and the two larger dyes is much more pronounced.

In the following paragraph, we will give an explanation of the observed differences in the relative changes of rotational and translational motion. The faster decrease in translational diffusion is due to entanglements, resulting in additional obstacles for translational diffusion. As the polymer concentration is increased more and more, entanglements occur and a network is formed. The entanglements are not permanent but reversibly formed since they are caused by physical intermolecular attractions rather than strong chemical bonds. Thus, the formation and cleavage of cross-links are constantly fluctuating. In the literature, mesh size with respect to the diffusant size has been extensively discussed in order to analyze diffusion in semidilute polymer solutions. ${ }^{52}$ During the course of polymerization the mesh size of the network is continuously decreased. As the mesh size approaches the size of the dyes, their motion is controlled not only by the macroviscosity of the solution but also by the dynamic structure of the meshes and thus the microviscosity. The relationship between probe size, length scale of the environment, macroviscosity, and microviscosity has been described by Szymański et al. ${ }^{53}$ With the additional influence of the meshes the relative changes in translational mobility will be more pronounced for larger than for smaller probes, a trend which is clear from Figure $3 \mathrm{~b}$. The difference between the diffusion coefficient of dye $\mathbf{1}$ and dye $\mathbf{3}$ normalized to its value in pure monomer solution can reach 1 order of magnitude.

In contrast to translation, relative changes in rotational motion show no significant size effects. For rotational motion to occur, the centroid of the dye does not have to change position. Thus, rotational motion is not significantly influenced by mesh size. Solely the increasing microviscosity, i.e., the increased friction due to the polymer chains around the probing dye, is responsible for the lowering of $D_{\mathrm{R}}$. Thus, rotational motion senses only the microviscosity of the solution whereas translational motion additionally yields information about mesh size.

The influence of permanent cross-links on the motion of dyes for different polymer concentrations has been discussed by Modesti et al., who compared a multiplicative and an additive contribution to the diffusion coefficient. ${ }^{25}$ By cross-linking radical polymerization, we introduced irreversible chemical crosslinks in addition to the entanglements which are also present in linear chains. This results in a decrease of the diffusion coefficients up to a factor of ca. 2 at conversions around $30 \%$. Permanent cross-links introduce additional and noncleavable obstacles for translational motion and cause significant heterogeneities in the system. ${ }^{54}$ In previous work we have shown by wide-field fluorescence microscopy measurements that permanent crosslinks even result in a fraction of immobile molecules. ${ }^{27}$ This fraction corresponds to molecules trapped in regions of high network density. In FCS measurements, however, this fraction remains obscured.

At high conversion we reach the glass transition which is at $x_{\mathrm{g}} \approx 0.8$ for these systems at room temperature as estimated by the Kelley-Bueche equation. ${ }^{55}$ Around $x_{\mathrm{g}}$, the diffusion coefficient drops by several orders of magnitude due to the well-known increase in viscosity. This drop is obvious in our data. However, since the low diffusion coefficients close to the glass transition are difficult to obtain by FCS, we do not have enough reliable data to draw conclusions about size effects on diffusion in this range. Thus, the effect of the glass transition will be the topic of future work.

\section{Conclusion}

In summary, we investigated the diffusion of dye molecules during the bulk radical polymerization of styrene by full 
correlation fluorescence correlation spectroscopy (fcFCS). Compared to earlier studies, this technique allows for the parallel detection of translation and rotational diffusion and thus for a direct comparison between them. Three different molecular probes were used to investigate the effects of probe size on molecular motion and to analyze how increasing restrictions during polymerization influence their motion. It was found that the small dye shows similar relative changes in the translational and rotational diffusion coefficient, whereas for the larger dyes additional restrictions in translational motion were observed. The results suggest that rotational motion is mainly influenced by microviscosity whereas translational motion additionally probes effects due to the mesh size of the entangled polymer chains. Differences between temporary and permanent cross-links were elucidated by comparison of a linear and a cross-linking radical polymerization.

Acknowledgment. Funding for this work was provided by the Zukunftskolleg of the Universität Konstanz. We thank Matthias Fuchs, Johan Hofkens, and Frans C. de Schryver for valuable discussions and Andrea Nagy for proofreading our manuscript.

Supporting Information Available: Determination of conversion by Raman spectroscopy. This material is available free of charge via the Internet at http://pubs.acs.org.

\section{References and Notes}

(1) Ediger, M. D. Annu. Rev. Phys. Chem. 1991, 42, 225-250.

(2) Neogi, P. Diffusion in Polymers; Marcel Dekker: New York, 1996.

(3) Wöll, D.; Braeken, E.; Deres, A.; De Schryver, F.; Uji-i, H.; Hofkens, J. Chem. Soc. Rev. 2009, 38, 313-328.

(4) Kulzer, F.; Xia, T.; Orrit, M. Angew. Chem., Int. Ed. 2010, 49, 854-866.

(5) Achilias, D. S. Macromol. Theory Simul. 2007, 16 (4), 319-347.

(6) Barner-Kowollik, C.; Russell, G. T. Prog. Polym. Sci. 2009, 34 (11), $1211-1259$.

(7) Russell, G. T. Aust. J. Chem. 2002, 55 (6-7), 399-414

(8) Barner-Kowollik, C.; Buback, M.; Egorov, M.; Fukuda, T.; Goto, A.; Olaj, O. F.; Russell, G. T.; Vana, P.; Yamada, B.; Zetterlund, P. B. Prog. Polym. Sci. 2005, 30 (6), 605-643.

(9) Tonge, M. P.; Gilbert, R. G. Polymer 2001, 42 (4), 1393-1405.

(10) Faldi, A.; Tirrell, M.; Lodge, T. P.; von Meerwall, E. Macromolecules 1994, 27, 4184-4192.

(11) Rauch, J.; Hartung, M.; Privalov, A. F.; Köhler, W. J. Chem. Phys. 2007, 126, 21

(12) Seiffert, S.; Oppermann, W. Polymer 2008, 49 (19), 4115-4126.

(13) Faldi, A.; Tirrell, M.; Lodge, T. P. Macromolecules 1994, 27, 41764183.

(14) Zielinski, J. M.; Heuberger, G.; Sillescu, H.; Wiesner, U.; Heuer, A.; Zhang, Y. M.; Spiess, H. W. Macromolecules 1995, 28 (24), 8287-8294

(15) Wisnudel, M. B.; Torkelson, J. M. Macromolecules 1996, 29 (19), 6193-6207.

(16) Zielinski, J. M.; Duda, J. L. AIChE J. 1992, 38 (3), 405-415.

(17) Phillies, G. D. J. J. Phys. Chem. 1989, 93 (13), 5029-5039.

(18) Masaro, L.; Zhu, X. X. Prog. Polym. Sci. 1999, 24 (5), 731-775.

(19) de Gennes, P.-G. Scaling Concepts in Polymer Physics; Cornell University Press: Ithaca, NY, 1979.

(20) Haustein, E.; Schwille, P. Annu. Rev. Biophys. Biomol. Struct. 2007, $36,151-169$.

(21) Rigler, R.; Elson, E. L. Fluorescence Correlation Spectroscopy. Theory and Applications; Springer-Verlag: Berlin, 2001.
(22) Zettl, H.; Zettl, U.; Krausch, G.; Enderlein, J.; Ballauff, M. Phys. Rev. E 2007, 75 (6), 061804

(23) Grabowski, C.; Mukhopadhyay, A. Macromolecules 2008, 41, 6191-6194.

(24) Zettl, U.; Hoffmann, S. T.; Koberling, F.; Krausch, G.; Enderlein, J.; Harnau, L.; Ballauff, M. Macromolecules 2009, 42 (24), 95379547.

(25) Modesti, G.; Zimmermann, B.; Borsch, M.; Herrmann, A.; Saalwächter, K. Macromolecules 2009, 42 (13), 4681-4689.

(26) Cherdhirankorn, T.; Harmandaris, V.; Juhari, A.; Voudouris, P.; Fytas, G.; Kremer, K.; Koynov, K. Macromolecules 2009, 42 (13), 4858-4866.

(27) Wöll, D.; Uji-i, H.; Schnitzler, T.; Hotta, J.; Dedecker, P.; Herrmann, A.; De Schryver, F. C.; Müllen, K.; Hofkens, J. Angew. Chem., Int. Ed. 2008, 47, 783-787.

(28) Zettl, H.; Hafner, W.; Böker, A.; Schmalz, H.; Lanzendorfer, M.; Müller, A. H. E.; Krausch, G. Macromolecules 2004, 37 (5), 19171920.

(29) Liu, R. G.; Gao, X.; Adams, J.; Oppermann, W. Macromolecules 2005, $38(21), 8845-8849$.

(30) Cherdhirankorn, T.; Best, A.; Koynov, K.; Peneva, K.; Müllen, K.; Fytas, G. J. Phys. Chem. B 2009, 113, 3355-3359.

(31) Cherdhirankorn, T.; Floudas, G.; Butt, H.-J.; Koynov, K. Macromolecules 2009, 42, 9183-9189.

(32) Omari, R. A.; Aneese, A. M.; Grabowski, C. A.; Mukhopadhyay, A. J. Phys. Chem. B 2009, 113 (25), 8449-8452.

(33) Rathgeber, S.; Beauvisage, H. J.; Chevreau, H.; Willenbacher, N.; Oelschlaeger, C. Langmuir 2009, 25 (11), 6368-6376.

(34) Widengren, J.; Mets, Ü.; Rigler, R. J. Phys. Chem. 1995, 99 (36), 13368-13379.

(35) Hanbury Brown, R.; Twiss, R. Q. Nature 1956, 177 (4497), 27-29.

(36) Felekyan, S.; Kühnemuth, R.; Kudryavtsev, V.; Sandhagen, C.; Becker, W.; Seidel, C. A. M. Rev. Sci. Instrum. 2005, 76, 8.

(37) Wahl, M.; Rahn, H.-J.; Gregor, I.; Erdmann, R.; Enderlein, J. Rev. Sci. Instrum. 2007, 78, 033106.

(38) Basché, T.; Moerner, W. E.; Orrit, M.; Talon, H. Phys. Rev. Lett. 1992, 69 (10), 1516-1519.

(39) Kask, P.; Piksarv, P.; Mets, Ü. Eur. Biophys. J. 1985, 12, 163-166.

(40) Ehrenberg, M.; Rigler, R. Chem. Phys. 1974, 4 (3), 390-401.

(41) Aragón, S. R.; Pecora, R. Biopolymers 1975, 14 (1), 119-137.

(42) Aragón, S. R.; Pecora, R. J. Chem. Phys. 1976, 64 (4), 1791-1803.

(43) Kask, P.; Piksarv, P.; Mets, Ü.; Pooga, M.; Lippmaa, E. Eur. Biophy. J. 1987, 14, 257-261.

(44) Kask, P.; Piksarv, P.; Pooga, M.; Mets, Ü.; Lippmaa, E. Biophys. J. 1989, 55, 213-220.

(45) Tsay, J. M.; Doose, S.; Weiss, S. J. Am. Chem. Soc. 2006, 128 (5), $1639-1647$

(46) Loman, A.; Gregor, I.; Stutz, C.; Mund, M.; Enderlein, J. Photochem. Photobiol. Sci. 2010, 2010 (9), 627-636.

(47) Qu, J. Q.; Zhang, J. Y.; Grimsdale, A. C.; Müllen, K.; Jaiser, F.; Yang, X. H.; Neher, D. Macromolecules 2004, 37 (22), 8297-8306.

(48) Kurtsiefer, C.; Zarda, P.; Mayer, S.; Weinfurter, H. J. Mod. Opt. 2001, 48, 2039-2047.

(49) Widengren, J.; Mets, Ü.; Rigler, R. Chem. Phys. 1999, 250 (2), 171-186.

(50) Müller, C. B.; Loman, A.; Pacheco, V.; Koberling, F.; Willbold, D.; Richtering, W.; Enderlein, J. Europhys. Lett. 2008, 83, 4.

(51) Lakowicz, J. R. Principles of Fluorescence Spectroscopy, 2nd ed.; Kluwer Academic/Plenum Publishers: New York, 1999.

(52) Tracy, M. A.; Garcia, J. L.; Pecora, R. Macromolecules 1993, 26 (8), $1862-1868$.

(53) Szymański, J.; Patkowski, A.; Wilk, A.; Garstecki, P.; Holyst, R. J. Phys. Chem. B 2006, 110 (51), 25593-25597.

(54) Guo, Z.; Sautereau, H.; Kranbuehl, D. E. Macromolecules 2005, 38 (19), 7992-7999.

(55) Mark, J. E. Physical Properties of Polymers Handbook, 2nd ed.; Springer: Berlin, 2007. 